\title{
INSETOS CAPTURADOS COM ARMADILHA MALAISE NA ILHA DO MEL, BAÍA DE PARANAGUÁ, PARANÁ, BRASIL. \\ II. TABANIDAE (DIPTERA) ${ }^{1}$
}

\author{
Renato R. C. Dutra ${ }^{2}$ \\ Renato C. Marinoni ${ }^{2}$
}

\begin{abstract}
Insects Captured with Malaise trap at the "Ilha do Mel", Baia de Paranaguá, Paraná, Brazil.. II. Tabanidae (Diptera). Two Malaise traps (TOWNES' 1972 model) were installed on an island (the "Ilha do Mel") in the Bay of Paranaguá, from September, 1988 to August, 1989, at two sites denominated "Fortaleza" and "Praia Grande", for collection of insects. At "Fortaleza", 1,715 individuals of Tabanidae were captured, representing $3.20 \%$ of all Diptera captured in this site, while at "Praia Grande" 1,420 individuals of the same family were captured, representing $5.06 \%$ of all Diptera captured there. In "Fortaleza", Tabanidae is represented for 10 species, while in "Praia Grande" it is represented for nine species. Dichelacera alcicomis (Wiedemann, 1828) was the most abundant species at two sites, representing $87.52 \%$ and $66.69 \%$ of all Tabanidae captured at "Fortaleza" and "Praia Grande", respectively. The greatest number of individuals occurred in November, the month with the lowest relative humidity associated to small pluviometric precipitation, just after a month with high relative humidity associated to a high pluviometric precipitation.

KEY WORDS. Tabanidae, phenology, Paraná
\end{abstract}

Os Tabanidae são dípteros popularmente conhecidos como "mutucas", principalmente do leigo que mora fora dos centros urbanos e dos veranistas que se deslocam para o litoral paranaense, os quais sofrem ataque e reações alérgicas decorrentes de suas doloridas picadas (FRANÇA 1975).

Anteriormente (MARINONI \& DUTRA 1993), definiram os objetivos do "Levantamento da Fauna Entomológica no Estado do Paraná", e mais especificamente na Ilha do Mel (Dutra \& MARINONI 1994). No presente trabalho, apresentamos o estudo a nível específico da família Tabanidae (Diptera), para a qual contamos com a identificação feita pelo Dr. Inocêncio S. Gorayeb (Museu Paraense Emílio Goeldi) e com uma boa coleção de referência existente na Coleção de Entomologia Pe. Jesus Santiago Moure, do Departamento de Zoologia da Universidade Federal do Paraná. A existência de um estudo anterior para o grupo, incluindo a região litorânea do Paraná (FRANÇA 1975), o depósito deste material na Coleção supracitada, a possibilidade de um estudo comparativo entre o material capturado na região continental e o do ambiente insular, além da facilidade de

1) Contribuição número 791 do Departamento de Zoologia, Universidade Federal do Paraná.

2) Departamento de Zoologia, Universidade Federal do Paraná, Caixa Postal 19020, 81531-970 Curitiba, Paraná, Brasil. Bolsista do CNPq. 
triagem do material, foram fatores preponderantes na escolha desta família.

Um dos objetivos do presente trabalho é avaliar o alcance da metodologia utilizada nas amostragens, para comparações dentro e entre ecossistemas (MARINONI \& DUTRA 1993).

\section{MATERIAL E MÉTODOS}

O material foi capturado em dois pontos da Ilha do Mel (Fortaleza e Praia Grande), com armadilha Malaise (modelo TowNES 1972), conforme descrito em DUTRA \& MARINONI (1994), onde também são encontrados os dados da área estudada e dos locais onde as armadilhas foram instaladas (situação florística e características geomorfológicas).

Os critérios utilizados para instalação das armadilhas encontram-se em MARINONI \& DUTRA (1993).

O período de amostragem incluiu 52 semanas, de setembro de 1988 a agosto de 1989, conforme as tabelas I e II.

Os dados meteorológicos (temperaturas máxima e mínima, umidade relativa do ar e precipitação), utilizados para comparação com os resultados da flutuação populacional dos Tabanidae, que foram expressos graficamente na forma de captura média (número médio de indivíduos/semana) (Tabs I e II), também podem ser encontrados em DUTRA \& MARINONI (1994).

\section{RESULTADOS E DISCUSSÃO}

\section{Sobre a QuANTIDAdE E a CAPTURA MÉdia da FAMILIA TABANIDAE E dE SUAS ESPÉCIES, CAPTURADAS NA FORTALEZA, NOS DIFERENTES MESES DO ANO}

Exemplares desta família foram capturados em sete dos doze meses estudados (não foram capturados em setembro de 1988 e de maio a agosto de 1989), com um total de 1.715 indivíduos (Tab. I), que representaram 3,20\% do total de Diptera capturado nesta localidade (DUTRA \& MARINONI 1994). A variação da captura média foi de 1,40 a 367,00 indivíduos/semana, registradas em abril e novembro respectivamente (Fig. 1).

Está representada pelas seguintes 10 espécies (Tab. I e Fig. 2):

\section{Catachlorops (Psalidia) furcatus (Wiedemann, 1828)}

Foi capturada em quatro dos doze meses estudados (outubro a dezembro e abril), com um total de 13 indivíduos, que representaram $0,76 \%$ do total de Tabanidae capturado nesta localidade (Tab. I). Apresentou uma variação da captura média de 0,25 a 1,20 indivíduos/semana, registradas em dezembro e outubro respectivamente (Fig. 2).

\section{Catachlorops fuscinevris (Macquart, 1838)}

Foi capturada apenas em novembro, com um total de 28 indivíduos $(7,00$ indivíduos/semana), que representaram 1,63\% do total de Tabanidae capturado nesta localidade (Tab. I e Fig. 2). 


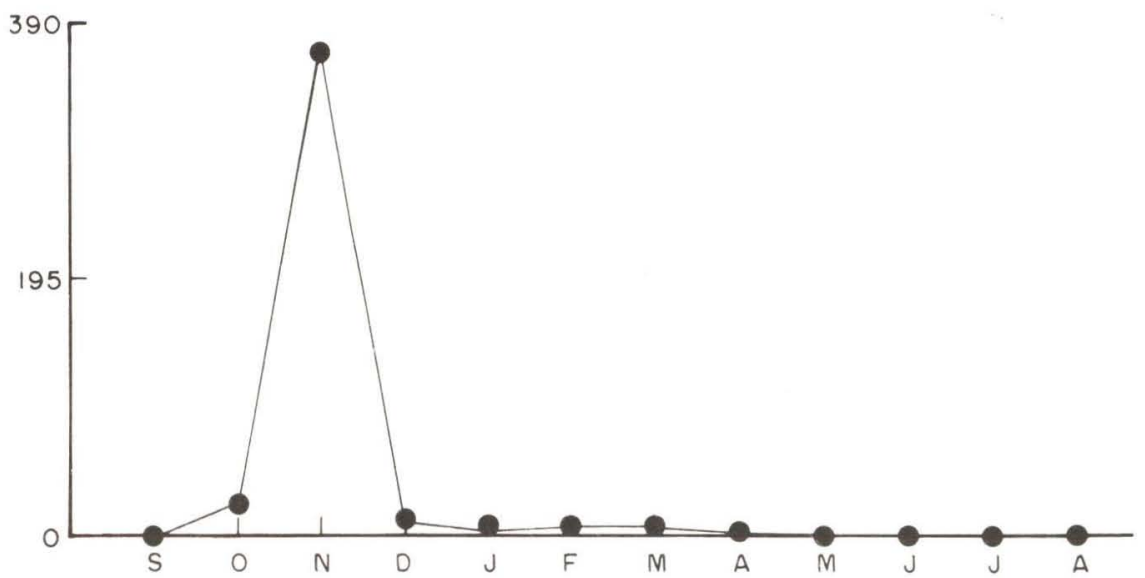

Fig. I. Ilha do Mel. Fortalèa. Flutuação anuál da captura media (numero medıo de individuos/semana) dos Tabanidae, com armadilha Malaise, no periodo de setembro de 1988 a agosto de 1989.

Tahela I. Ilta do Mel. Fortaleza. Tahanidae. Número total de individuos, por espécie, capturados com armadilha Malaise. no periodo de setembro de 1988 a agosto de 1989.

\begin{tabular}{lccccccccccccc}
\hline \multicolumn{1}{c}{ ESPECIES } & SET & $(88)$ & OUT NOV & DEZ JAN $(89)$ & FEV & MAR & ABR & MA! & JUN & JUL & AGO TOTAL \\
\hline Catachlorops furcatus & 0 & 6 & 2 & 1 & 0 & 0 & 0 & 4 & 0 & 0 & 0 & 0 & 13 \\
Catachlorops fuscinevris & 0 & 0 & 28 & 0 & 0 & 0 & 0 & 0 & 0 & 0 & 0 & 0 & 28 \\
Chlorotabanus inanis & 0 & 0 & 0 & 0 & 0 & 0 & 0 & 0 & 0 & 0 & 0 & 0 & 0 \\
Chrysops sp. & 0 & 0 & 0 & 1 & 0 & 0 & 0 & 0 & 0 & 0 & 0 & 0 & 1 \\
Diachiorus bivitatus & 0 & 0 & 42 & 6 & 2 & 3 & 17 & 0 & 0 & 0 & 0 & 0 & 70 \\
Dichelacera alcicornis & 0 & 115 & 1362 & 24 & 0 & 0 & 0 & 0 & 0 & 0 & 0 & 0 & 1501 \\
Phaeotabanus litigiosus & 0 & 0 & 0 & 1 & 0 & 0 & 0 & 0 & 0 & 0 & 0 & 0 & 1 \\
Poeciloderas sp. & 0 & 0 & 3 & 0 & 0 & 0 & 0 & 0 & 0 & 0 & 0 & 0 & 3 \\
Pseudacanthocera sylverii & 0 & 0 & 0 & 1 & 0 & 0 & 0 & 0 & 0 & 0 & 0 & 0 & 1 \\
Stenotabanus sp. & 0 & 0 & 19 & 6 & 2 & 22 & 11 & 1 & 0 & 0 & 0 & 0 & 61 \\
Tabanus occidentalis & 0 & 0 & 12 & 9 & 10 & 2 & 1 & 2 & 0 & 0 & 0 & 0 & 36 \\
\hline TOTAL & 0 & 121 & 1468 & 49 & 14 & 27 & 29 & 7 & 0 & 0 & 0 & 0 & 1715 \\
\hline No DE AMOSTRAS & 4 & 5 & 4 & 4 & 5 & 4 & 4 & 5 & 4 & 4 & 5 & 4 & 52 \\
\hline
\end{tabular}

\section{Chrysops sp.}

Foi capturada apenas na Fortaleza, em dezembro, com um único indivíduo ( 0,25 indivíduos/semana), que representou $0,06 \%$ do total de Tabanidae capturado nesta localidade (Tab. I e Fig. 2).

4. Diachlorus hivitatus (Wiedemann, 1828)

Foi capturada em cinco dos doze meses estudados (novembro a março), com um total de 70 indivíduos, que representaram 4,08\% do total de Tabanidae capturado nesta localidade (Tab. I). Apresentou uma variação da captura média 
de 0,40 a 10,50 indivíduos/semana, registradas em janeiro e novembro respectivamente (Fig. 2).

C. furcotus

C. fuscinevris

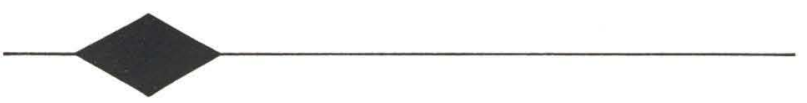

Chrysops sp

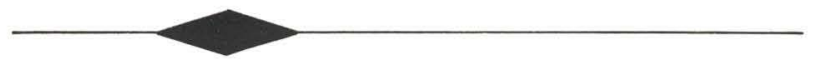

D. bivitatus

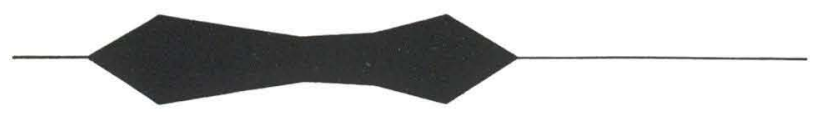

D. alcicornis

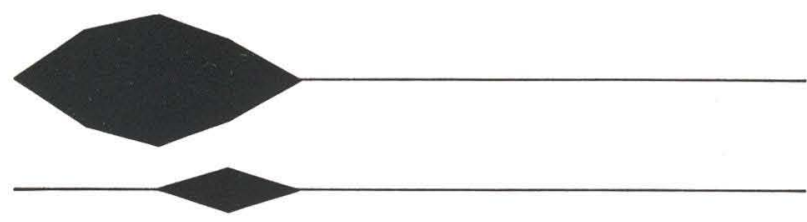

Poeciloderos sp

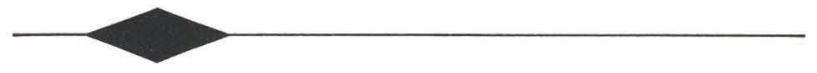

P. sylverii

Stenotabanus sp

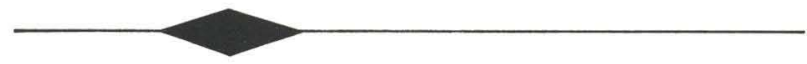

I. occidentalis
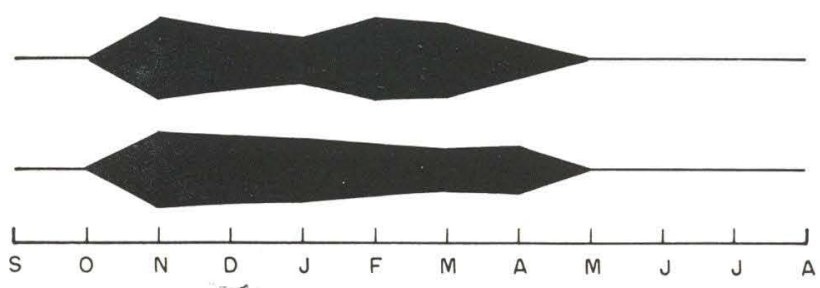

Fig. 2. Ilha do Mel. Fortaleza. Flutuaçào anual da captura média (número médio de indivíduos/semana) das dez espécies de Tabanidae, com armadilha Malaise, no período de setembro de 1988 a agosto de 1989.

5. Dichelacera alcicornis (Wiedemann, 1828)

Foi capturada em três dos doze meses estudados (outubro a dezembro), com um total de 1.501 indivíduos, que representaram $87,52 \%$ do total de Tabanidae capturado nesta localidade (Tab. I). Apresentou uma variação da captura média de 6,00 a 340,50 indivíduos/semana, registradas em dezembro e novembro respectivamente (Fig. 2).

6. Phacotabanus litigiosus (Walker, 1850)

Foi capturada apenas na Fortaleza, em dezembro, com um único indivíduo 
(0,25 individuos/semana), que representou $0,06 \%$ do total de Tabanidae capturado nesta localidade (Tab. I e Fig. 2).

\section{Poeciloderas sp.}

Foi capturada apenas no mês de novembro, com um total de três indivíduos $(0,75$ indivíduos/semana $)$, que representaram $0,17 \%$ do total de Tahanidae capturado nesta localidade (Tab. I e Fig. 2).

8. Pseudacanthocera sylverii (Macquart, 1838)

Foi capturada apenas em dezembro, com um único indivíduo $(0,25$ indivíduos/semana), que representou $0.06 \%$ do total de Tabanidae capturado nesta localidade (Tah. I e Fig. 2).

9. Stenotahamus (Stenotahanus) sp.

Foi capturada em seis dos doze meses estudados (novembro a abril), com um total de 61 indivíduos, que representaram $3,56 \%$ do total de Tabanidae capturado nesta localidade (Tah. I). Apresentou uma variação da captura média de 0,20 a 5,50 indivíduos/semana, registradas em abril e fevereiro respectivamente (Fig. 2).

\section{Tabamus occidentalis Linnaeus, 1758}

Foi capturada em seis dos doze meses estudados (novembro a abril), com um total de 36 indivíduos, que representaram 2,10\% do total de Tabanidae capturado nesta localidade (Tah. I). Apresentou uma variação da captura média de 0,25 a 3,00 indivíduos/semana, registradas em março e novembro respectivamente (Fig. 2).

\section{Sobre a QUANTIDAdE E A CAPTURA MÉdIA DA FAMILIA TABANIDAE E dE SUAS ESPÉCIES, CAPTURAdAS NA PRAIA GRANDE, NOS DIFERENTES MESES DO ANO}

Exemplares desta família foram capturados em oito dos doze meses estudados (não foram capturados de maio a agosto de 1989), com um total de 1.420 indivíduos (Tab. II) que representaram 5,06\% do total de Diptera capturado nesta localidade (DUTRA \& MARINONI 1994). A variação da captura média foi de 0,25 a 277,50 indivíduos/semana, registradas em setembro e novembro respectivamente (Fig. 3).

Está representada pelas seguintes nove espécies (Tab. II e Fig. 4):

\section{Catachlorops (Psalidia) furcatus (Wiedemann. 1828)}

For capturada em quatro dos doze meses estudados (outubro a dezembro e março), com um total de 96 indivíduos, que representaram 6,76\% do total de Tahanidae capturado nesta localidade (Tah. II). Apresentou uma variação da captura média de 0,25 a 12,50 indivíduos/semana, registradas em março e novembro respectivamente (Fig. 4).

\section{Catachlorops fuscinevris (Macquart, 1838)}

Foi capturada em dois dos doze meses estudados (novembro e dezembro), com um total de 53 indivíduos, que representaram 3,73\% do total de Tabanidae capturado nesta localidade (Tah. II). Apresentou uma variação da captura média 


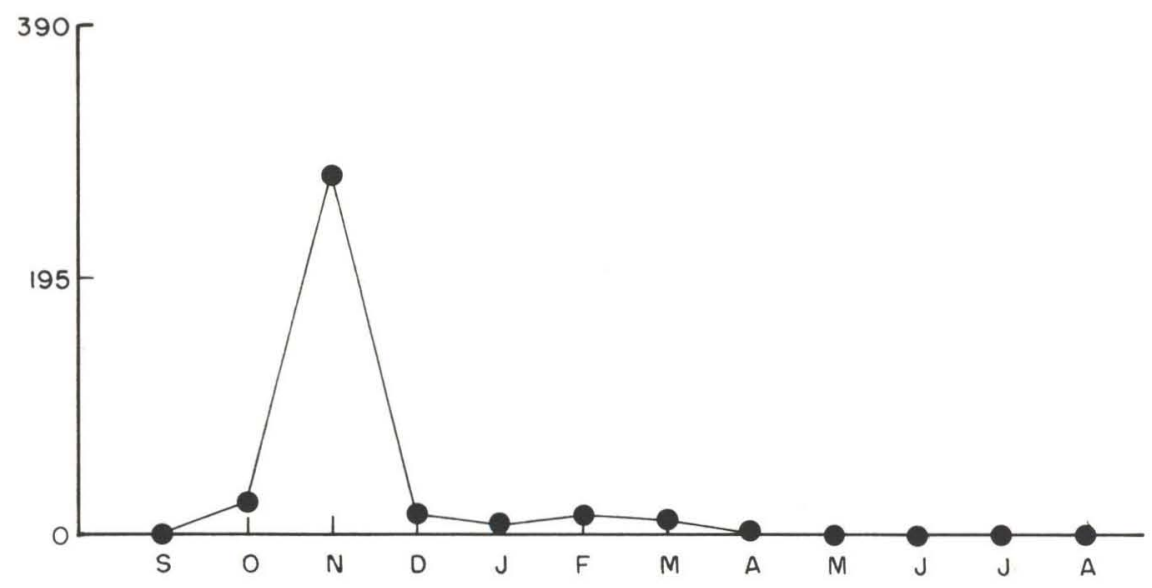

Fig. 3. Ilha do Mel. Praia Grande. Flutuação anual da captura média (número médio de indivíduos/semana) dos Tabanidae, com armadilha Malaise, no período de setembro de 1988 a agosto de 1989.

Tabela II. Ilha do Mel. Praia Grande. Tabanidae. Número total de indivíduos, por espécie, capturados com armadilha Malaise, no período de setembro de 1988 a agosto de 1989.

\begin{tabular}{lcrrrrrrrrrrrrr}
\hline \multicolumn{1}{c}{ ESPÉCIES } & SET (88) & OUT & NOV & DEZ JAN & (89) & FEV & MAR & ABR & MAI & JUN & JUL & AGO TOTAL \\
\hline Catachlorops furcatus & 0 & 39 & 50 & 6 & 0 & 0 & 1 & 0 & 0 & 0 & 0 & 0 & 96 \\
Catachlorops fuscinevris & 0 & 0 & 52 & 1 & 0 & 0 & 0 & 0 & 0 & 0 & 0 & 0 & 53 \\
Chlorotabanus inanis & 0 & 0 & 0 & 1 & 0 & 0 & 0 & 0 & 0 & 0 & 0 & 0 & 1 \\
Chrysops sp. & 0 & 0 & 0 & 0 & 0 & 0 & 0 & 0 & 0 & 0 & 0 & 0 & 0 \\
Diachlarus bivitatus & 0 & 0 & 8 & 2 & 1 & 0 & 0 & 0 & 0 & 0 & 0 & 0 & 11 \\
Dichelacera alcicornis & 0 & 57 & 886 & 4 & 0 & 0 & 0 & 0 & 0 & 0 & 0 & 0 & 47 \\
Phaeotabanus litigiosus & 0 & 0 & 0 & 0 & 0 & 0 & 0 & 0 & 0 & 0 & 0 & 0 & 0 \\
Poeciloderas sp. & 0 & 7 & 5 & 2 & 1 & 0 & 0 & 0 & 0 & 0 & 0 & 0 & 15 \\
Pseudacanthocera syiverii & 0 & 0 & 0 & 0 & 1 & 0 & 0 & 0 & 0 & 0 & 0 & 0 & 1 \\
Stenotabanus sp. & 0 & 0 & 52 & 10 & 3 & 38 & 27 & 9 & 0 & 0 & 0 & 0 & 39 \\
Tabanus occidentalis & 0 & 20 & 57 & 32 & 22 & 19 & 6 & 0 & 0 & 0 & 0 & 0 & 157 \\
\hline TOTAL & 0 & 123 & 1110 & 58 & 28 & 57 & 34 & 9 & 0 & 0 & 0 & 0 & 1420 \\
\hline No DE AMOSTRAS & 4 & 5 & 4 & 4 & 5 & 4 & 4 & 5 & 4 & 4 & 5 & 4 & 52 \\
\hline
\end{tabular}

de 0,25 a 13,00 indivíduos/semana, registradas em dezembro e novembro respectivamente (Fig. 4).

3. Chlorotabanus inanis (Fabricius, 1797)

Foi capturada apenas na Praia Grande, em dezembro, com um único indivíduo $(0,25$ indivíduos/semana), que representou $0,07 \%$ do total de Tabanidae capturado nesta localidade (Tab. II e Fig. 4). 
C. furcotus

C. fuscinevris

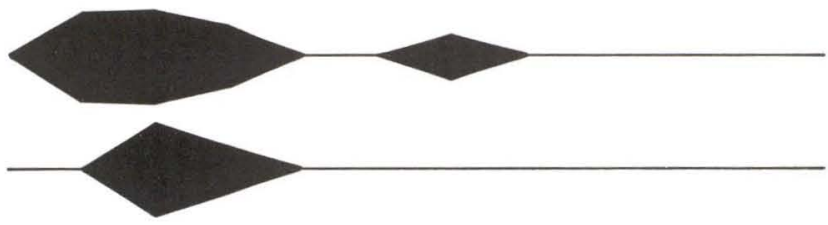

C. inanis

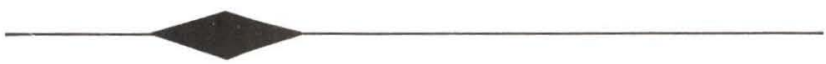

D. bivitatus

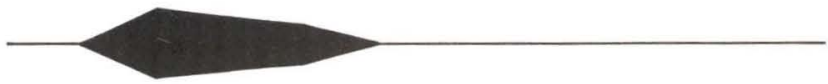

D. alcicornis

Poeciloderos sp

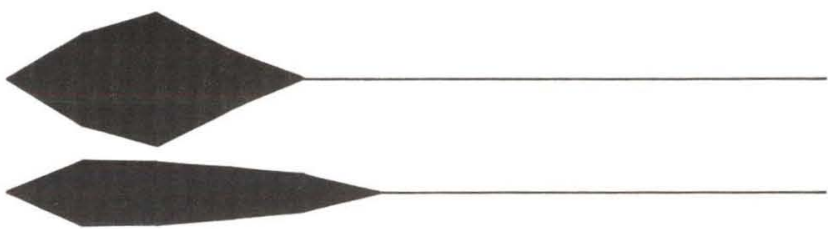

P. sylverii

Stenotobanus Sp

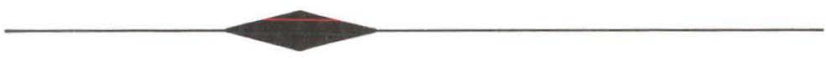

I. occidentalis
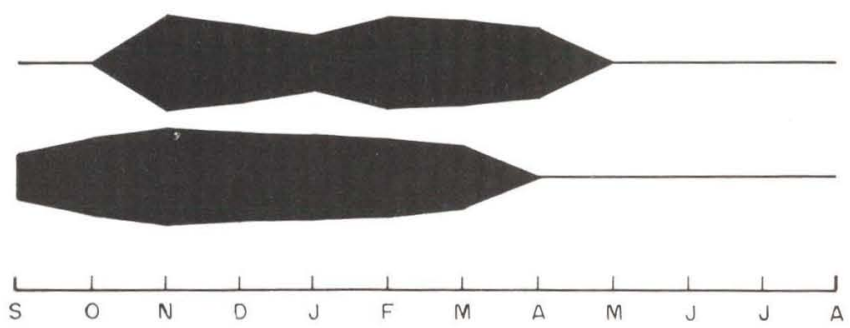

Fig. 4. Itha de Mel. Prata Grande. Flutuayas anual da eaptura média (número médue de individuos/semana) das nove espécies de Tahanidae. eom armadilha Malaise. no período de setembro de 1988 a agosto de 1989.

\section{Diachlorus bivitatus (Wiedemann, 1828)}

Foi capturada em três dos doze meses estudados (novembro a janeiro), com um total de 11 indivíduos, que representaram $0,77 \%$ do total de Tabanidae capturado nesta localidade (Tab. II). Apresentou uma variação da captura média de 0,20 a 2,00 indivíduos/semana, registradas em janeiro e novembro respectivamente (Fig. 4).

\section{Dichelacera alcicornis (Wiedemann, 1828)}

Foi capturada em três dos doze meses estudados (outubro a dezembro), com um total de 947 indivíduos, que representaram 66,69\% do total de Tabanidae capturado nesta localidade (Tab. II). Apresentou uma variação da captura média de 1,00 a 221,50 indivíduos/semana, registradas em dezembro e novembro respectivamente (Fig. 4). 


\section{Poeciloderas sp.}

Foi capturada em quatro dos doze meses estudados (outubro a janeiro), com um total de 15 indivíduos, que representaram $1,06 \%$ do total de Tabanidae capturado nesta localidade (Tab. II). Apresentou uma variação da captura média de 0,20 a 1,40 indivíduos/semana, registradas em janeiro e outubro respectivamente (Fig. 4).

\section{Pseudacanthocera sylverii (Macquart, 1838)}

Foi capturada apenas em janeiro, com um único indivíduo $(0,20$ indivíduos/semana), que representou $0,07 \%$ do total de Tabanidae capturado nesta localidade (Tab. II e Fig. 4).

\section{Stenotabanus (Stenotabanus) sp.}

Foi capturada em seis dos doze meses estudados (novembro a abril), com um total de 139 indivíduos, que representaram 9,79\% do total de Tabanidae capturado nesta localidade (Tab. II). Apresentou uma variação da captura média de 0,75 a 13,00 indivíduos/semana, registradas em janeiro e novembro respectivamente (Fig. 4).

\section{Tabanus occidentalis Linnaeus, 1758}

Foi capturada em sete dos doze meses estudados (setembro a março), com um total de 157 indivíduos, que representaram 11,06\% do total de Tabanidae capturado nesta localidade (Tab. II). Apresentou uma variação da captura média de 0,25 a 14,25 indivíduos/semana, registradas em setembro e novembro respectivamente (Fig. 4).

As 11 espécies de Tabanidae capturadas na Ilha do Mel representam um número bem menor que as 23 espécies capturadas por FRANÇA (1975), na região litorânea do Estado do Paraná, o qual utilizou equídeos como atrativos durante as amostragens.

Segundo ROBERTS $(1970,1971)$ a cor e a localização da armadilha Malaise podem afetar a captura de Tabanidae, sugerindo a utilização de atrativos $\left(\mathrm{CO}_{2}\right.$, por exemplo) para detectar as espécies que ocorrem em pequeno número e/ou por períodos relativamente curtos.

Outra possibilidade foi postulada por MACARTHUR \& WILSON (apud CurE-HAKIM 1983), segundo a qual a distância entre uma ilha e a fonte de espécies, assim como a área da ilha, são fatores que influenciam a diversidade; a área raramente exercendo um efeito direto sobre a presença de uma espécie mas determinando, geralmente, uma maior variabilidade de habitats, os quais influem na ocorrência das espécies (LANZER \& SCHAFER 1987).

Se por um lado SoUTHWOOD (1961) demonstra que a grande maioria dos Insecta, até mesmo Collembola, são carregados pelas correntes aéreas por grandes distâncias, BERRY (1983) confirma a tendência das ilhas de possuírem um menor número de espécies que áreas continentais, de dimensões similares. Segundo ZIMMERMAN \& BIERREGAARD (1986) a relação espécies-área tem promovido as bases teóricas para o desenho das reservas naturais, podendo predizer a área mínima necessária para preservar um certo número de espécies (SULLIVAN \& 
SHAFFER 1975; DiAMOND 1976; SimberlofF \& ABElE 1976 e LEWIN 1984, entre outros).

FRANCA (1975) observou que, durante o inverno no litoral, a atividade dos Tabanidae cessou, apesar de registrar que a temperatura muito alta também atuou como fator limitante da atividade. Os resultados do presente trabalho também apresentam esta tendência, sendo importante salientar que o grande número de indivíduos registrados em novembro (2.578 indivíduos $-82,23 \%$ do total de Tabanidae capturado para as duas localidades simultaneamente) ocorreu no mês de menor umidade relativa média associada a uma baixa precipitação pluviométrica (DUTRA \& MARINONI 1994), e que se seguiu a um mês de alta umidade relativa média associada a uma alta precipitação.

Três espécies, comprovadamente, foram comuns aos dois trabalhos em questão: Diachlorus bivitatus, Dichelacera alcicornis e Phaeotabanus litigiosus; as freqüências percentuais e os meses de maior atividade destas espécies diferindo significativamente entre os dois trabalhos, sugerindo a existência de características estruturais próprias das comunidades insulares. Corrobora esta observação a pouca representatividade de Dichelacera alcicornis nas amostras de FRANCCA (1975), enquanto esta foi a espécie mais abundante no ambiente insular.

De imediato dois fatos chamam a atenção. Inicialmente, o número de exemplares de Tabanidae, capturados nas duas localidades da Ilha do Mel, é bastante semelhante, apesar do número de Diptera capturados na Fortaleza ser praticamente o dobro daquele obtido para a Praia Grande (DUTRA \& MARINONI 1994). Em segundo lugar, as flutuações das capturas médias dos Tabanidae e de suas espécies mais representativas apresentaram a mesma tendência nas duas localidades, sendo notável as semelhanças observadas quando comparados os meses em que Catachlorops furcatus, Catachlorops fuscinevris, Dichelacera alcicornis e Stenotabanus sp. foram capturadas. Estas coincidências confirmam a eficiência da armadilha Malaise para comparações dentro de um mesmo ecossitema.

\section{REFERÊNCIAS BIBLIOGRÁFICAS}

BERRY, R.J. 1983. Diversity and differentiation: the importance of island biology for general theory. Oikos 41: 523-529.

CURE-HAKIM, J.R. 1983. Estudo ecoloógico da comunidade de abelhas silvestres (Hymenoptera, Apoidea) do Parque da Cidade, comparado ao de outras áreas de Curitiha, Paraná. Tese de Mestrado, não publicada, Universidade Federal do Paraná, Curitiba, 96p.

Diamond, J.M. 1976. Island Biogeography and Conservation: Strategy and Limitations. Science 193: 1027-1029.

DUtRA, R.R.C. \& R.C. MARINONI. 1994. Insetos capturados com armadilha Malaise na Ilha do Mel, Baía de Paranaguá, Paraná, Brasil. I. Composição ordinal. Revta bras. Zool. 11 (2): 227-245.

FrançA, J.M. 1975. Sobre o comportamento de alguns tahanídeos do litoral 
e do primeiro planalto do Estado do Paraná, Brasil (Diptera, Tabanidae). Tese de Mestrado, não publicada, Universidade Federal do Paraná, Curitiba, $63 p$.

LANZER, R.M. \& A.E. SCHAFER. 1987. Moluscos dulceaquícolas como indicadores de condições tróticas em Lagoas costeiras do Sul do Brasil. Rev. Brasil. Biol. 47 (1/2): 47-56.

LEWIN, R. 1984. Parks: How big is big enough? Science 225: 611-612.

MARINONI, R.C. \& R.R.C. DUTRA. 1993. Levantamento da fauna entomológica no Estado do Paraná. I. Introdução. Situação climática e florística dos oito pontos de coleta. Dados faunísticos de agosto de 1986 a julho de 1987. Revta bras. Zool. 8 (1-4): 31-73.

RoberTs, R.H. 1970. Color of Malaise traps and the collection of Tabanidae. Mosquito News 30 (4): 567-571.

1971. The seasonal appearance of Tabanidae as determined by Malaise trap collections. Mosquito News 31 (4): 509-512.

SimBERLofF, D.S. \& L.G. ABELE. 1976. Island Biogeography Theory and Conservation Practice. Science 191: 285-286.

SouthwOOD, T.R.E. 1961. The number of species of insect associates with various trees. J. Anim. Ecol. 30: 1-8.

Sullivan, A.L. \& M.L. Shaffer. 1975. Biogeography of the Megazoo. Science 189: $13-17$.

Townes, H. 1972. A light-weiht Malaise trap. Ent. News 83: 239-247.

Zimmerman, B.L. \& R.O. BierRegaARD. 1986. Relevance of the equilibrium theory of island biogeography and species-area relations to conservation with a case from Amazonia. J. Biogeogr. 13: 133-143.

Recebido em 25.I.1994; aceito em 26.IV.1994. 